\title{
IMPLEMENTACIÓN Y EVALUACIÓN DEL PROGRAMA DE EDUCACIÓN PATRIMONIAL "PEPA"
}

Gianina Sánchez Vega
Desiree Román Ponce ${ }^{(*)}$

PEPA es el Programa de Educación Patrimonial que nace de la preocupación del Consejo de Monumentos Nacionales de Chile $^{1}(\mathrm{CMN})$, por aportar al conocimiento y difusión del patrimonio declarado bajo la Ley $17.288,{ }^{2}$ dentro del ámbito escolar y educativo en general.

La iniciativa se desarrolló y elaboró por la, entonces, Área de Educación y Difusión del CMN durante los años 2011-2015, recibiendo financiamiento del Fondo de Acciones Culturales Complementarias de la DIBAM, ${ }^{3}$ en la línea de Proyectos Patrimoniales y aportes de la institución.

El patrimonio, tanto cultural como natural, es una temática transversal que puede aportar en el proceso de enseñanza-aprendizaje de las distintas áreas del conocimiento. Sus extraordinarios valores y los diversos ámbitos que abarca le otorgan potencial para desarrollar metodologías y actividades únicas, innovadoras y muchas veces multi e interdisciplinarias.

PEPA tiene como principal objetivo fomentar, en la comunidad escolar, la valoración del patrimonio cultural y natural, apoyando las líneas del curriculum escolar. Con ello se espera aportar al fortalecimiento de la identidad local y nacional, así como el aprecio de la diversidad cultural.

Otorga a las y los docentes herramientas para la utilización del patrimonio cultural y natural, a través de la investigación de sus posibilidades pedagógicas identificando otras vías de incorporación en el currículo escolar y generando capacidades para diseñar actividades didácticas que involucren al patrimonio como medio para introducir conocimiento de un modo colaborativo y experiencial.

\footnotetext{
${ }^{(*)}$ Gianina Sánchez Vega. Consejo de Monumentos Nacionales, Chile. E-mail: gsanchez@ monumentos.cl.

Desiree Román Ponce. Museo Nacional de Historia Natural, Chile. Magister en Desarrollo Curricular y Proyectos Educativos de la Universidad Andrés Bello E-mail: desiree.roman@mnhn.cl.

${ }^{1}$ Organismo técnico del Estado de Chile, que depende del Ministerio de Educación y desde su creación en 1925, se encarga de la protección y tuición del patrimonio de carácter monumental. (http://www.monumentos.cl/onsejo/ 606/w3channel.html).

${ }^{2}$ Legislación sobre Monumentos Nacionales de Chile.

3 Dirección de Bibliotecas, Archivos y Museos (Dibam), institucionalidad cultural del Estado de Chile se ha desarrollado al alero del Ministerio de Educación.
} 
Fomenta entre los niñ@s y jóvenes, el reconocimiento y valoración del patrimonio natural y cultural del país declarado (bajo la Ley 17.288), apreciando la importancia de los bienes patrimoniales como una construcción social en permanente cambio.

Con ese fin, PEPA propone e implementa diversos recursos de apoyo y actividades complementarias que acercan a la comunidad escolar a la riqueza cultural del país.

Al comenzar nuestro trabajo nos planteamos varias interrogantes, entre ellas ¿En qué nivel educativo sería más interesante y significativo vincular la temática patrimonial?, para ello revisamos los programas educativos vigentes en la educación Chilena y nos quedamos con la primera infancia. Es en este período donde; por la flexibilidad curricular, las características y las etapas de desarrollo en que se encuentran sus alumnos y alumnas nos permitía integrar la temática en diferentes ámbitos de aprendizaje.

Al revisar las bases curriculares de la educación parvularia del Ministerio de Educación ${ }^{4}$ (Mineduc) descubrimos que sus objetivos y sus ámbitos de aprendizajes son muy adecuados para poder materializar el proyecto. Por lo cual logramos establecer espacios de vinculación de la educación patrimonial con los tres grandes ámbitos de experiencias.

1. Ámbito Formación Personal y Social.

2. Ámbito de la Comunicación.

3. Ámbito de la Relación con el Medio Natural y Cultural.

En su conjunto, éstos abarcan campos de acción donde se llevan a cabo procesos claves para la tarea formativa y de aprendizaje de los párvulos.

En todos y cada uno de ellos es posible incluir la temática patrimonial ya que es transversal e instrumental para otros aprendizajes. Sin embargo en los ámbitos de Formación Personal - Social y Relación con el Medio Natural - Cultural, es donde se puede incorporar con mayor atingencia. Es en esta etapa del desarrollo donde, los niños y niñas, acercan por primera vez a los conceptos de identidad y pertenencia para así lograr aprendizajes significativos como lo plantean Hevia, Hirmas, Peñafiel,

Para que los aprendizajes sean significativos tienen que vincularse con las experiencias de vida que sean previamente importantes y valiosas para los alumnos; esto es, deben vincularse con su "patrimonio cultural" y tener, como punto de partida, los valores de la familia y el contexto cultural de la comunidade. (Hevia, Hirmas, Peñafiel 2002, p. 11),

\footnotetext{
${ }^{4}$ Fonte: <http://www.docentemas.cl/docs/2014/Educacion\%20Parvularia/Bases\%20curriculares\%20de\%201a\%20 Educacion\%20Parvularia.pdf $>$.
} 
saber quién es, conocer su comunidad y establecer lazos con ella son los elementos fundamentales para el desarrollo integral del niño y la niña, desarrollar habilidades y actitudes necesarias para comprender la vida en sociedad es fundamental para enfrentar los nuevos desafíos del mundo globalizado.

Debemos mencionar que en el curriculum escolar chileno no existe una asignatura específica dedicada a la educación patrimonial, todas las acciones en este ámbito se realizan desde la educación no formal, además en sus niveles tempranos hay poco material para trabajar en patrimonio, es que se vio una línea importante de acción, considerando los beneficios que este tiene para el proceso educativo, ya que

El patrimonio es una producción cultural a partir de la cual podemos movilizar una acción pedagógica dirigida a fortalecer la identidad, el sentido de pertenencia, el aprecio por lo propio recibido como herencia y la capacidad para hacer de esa herencia un factor de enriquecimiento personal y colectivo, y de progreso material y moral de las sociedades. (SÁNCHEZ, 1998, p. 43) es en este marco que el Consejo de Monumentos Nacionales propone a PEPA.

Como podemos apreciar la educación patrimonial es pertinente en la educación parvularia ya que nos permite intervenir en los diferentes ámbitos de aprendizajes con actividades no formales ya que ambas poseen, en su concepción, un curriculum flexible donde es posible realizar adecuaciones.

Ley General de la Educación chilena en su artículo $n^{\circ} 2$ establece:

La educación es el proceso de aprendizaje permanente de las personas que tiene como finalidad alcanzar su desarrollo espiritual, ético, moral, afectivo, intelectual, artístico y físico, mediante la transmisión y el cultivo de valores, conocimientos y destrezas.

Se enmarca en el respeto y valoración de los derechos humanos y de las libertades fundamentales, de la diversidad multicultural y de la paz, y de nuestra identidad nacional, capacitando a las personas para conducir su vida en forma plena, para convivir y participar en forma responsable, tolerante, solidaria, democrática y activa en la comunidad, y para trabajar y contribuir al desarrollo del país. (Ley 20.370 o LGE Ley general de educación 12 de septiembre 2009).

Por lo cual la educación patrimonial es la herramienta fundamental para que se cumpla el objetivo de la ley General de Educación de nuestro país, más aun si definimos

educación patrimonial como la acción educativa consciente, organizada y sistematizada dirigida a la formación de sujetos a partir del reconocimiento y la apropiación de su sustento cultural, histórico, político y ético-espiritual. Es decir, a partir del reconocimiento de su particularidad y de la apropiación plena, subjetiva y emancipatoria de su cultura, entendida ésta como un complejo sistema de valores, creencias, tradiciones, costumbres y horizontes utópicos 
constitutivo y constituyente de bienes materiales y espirituales únicos, irrepetibles e históricamente determinados. (CANTÓN, 2009, p. 5).

En los últimos años ha habido un incremento de proyectos educativos que se han ido incorporando a la gestión del patrimonio cultural buscando generar, en los ciudadanos, procesos de apropiación de los bienes culturales como parte de su propia identidad. En este sentido, el Consejo de Monumentos Nacionales de Chile (CMN) propone su programa de educación patrimonial PEPA.

PEPA espera alentar a los y las profesores (as) de distintas disciplinas a trabajar y crear nuevas iniciativas para infundir en los y las estudiantes el deseo de conocer, apropiarse y actuar en favor de la valoración y protección del patrimonio de nuestro país. Este programa aporta una propuesta didáctica materializada en un material concreto dirigido principalmente a la enseñanza inicial, entregando a los educadores una visión integrada del patrimonio permitiéndoles incorporar, a su quehacer, las temáticas patrimoniales en distintos ámbitos del aprendizaje que el currículum les exige. Su fácil modo de uso, su flexibilidad y su potente tópico permiten utilizarlo también en los niveles del primer ciclo básico trabajando distintas asignaturas tales como; historia, artes, ciencias, tecnología, lenguaje, entre otros, debido a sus características como:

UNIVERSALIDAD. El patrimonio es un elemento común y reconocido por todos que ayuda a desarrollar aprendizajes significativos al promover sentimientos de pertenencia en los grupos y en el desarrollo personal de los estudiantes.

PARTICIPACIÓN. Ayuda a la escuela a vincularse con la comunidad ya que potencia las relaciones entre el centro educativo y su entorno.

INTERCULTURIDAD. El patrimonio se constituye a partir de la diversidad, es una herramienta que otorga dinamismo, variedad y creatividad en la práctica docente.

INTEGRALIDAD. El patrimonio ayuda a crear un buen clima en el aula, ya que permite reconocernos, entendernos y respetarnos en la diversidad.

CALIDAD. El patrimonio reúne los valores, creencias, y manifestaciones de nuestra cultura y favorece la creación de una metodología pedagógica activa e interdisciplinar que ayuda a desarrollar en los alumnos competencias básicas.

\section{EL PROGRAMA}

El programa se basa en el despliegue de acciones pedagógicas formales, no formales e informales que integran la perspectiva de distintas disciplinas y utiliza una metodología interactiva, 
con el fin de fomentar instancias de reflexión, investigación y desarrollo creativo en los escolares y docentes. Las acciones pedagógicas se materializan en tres estrategias:

a) Desarrollo de una Plataforma Web <www.aprendeconpepa.cl>, dirigida a la comunidad escolar. Esta tiene como objetivo facilitar el aprendizaje interactivo en torno al patrimonio cultural y natural del país, así como la difusión de los Monumentos Nacionales y su valor cultural. Este sitio cuenta con un personaje protagonista llamado PEPA, que tiene la misión de acompañar la navegación y el aprendizaje de niños y niñas en un viaje por el territorio chileno, permitiendo la visualización de información descriptiva y audiovisual acerca de los Monumentos Nacionales en sus diferentes categorías. Este además presenta cuatro secciones temáticas o mini-sitios: patrimonio paleontológico, arquitectónico, arqueológico e histórico. Se busca con ello facilitar el aprendizaje interactivo y colectivo de los escolares en la web a través de aplicaciones: juegos en línea, descargas de información, guías didácticas, etc.

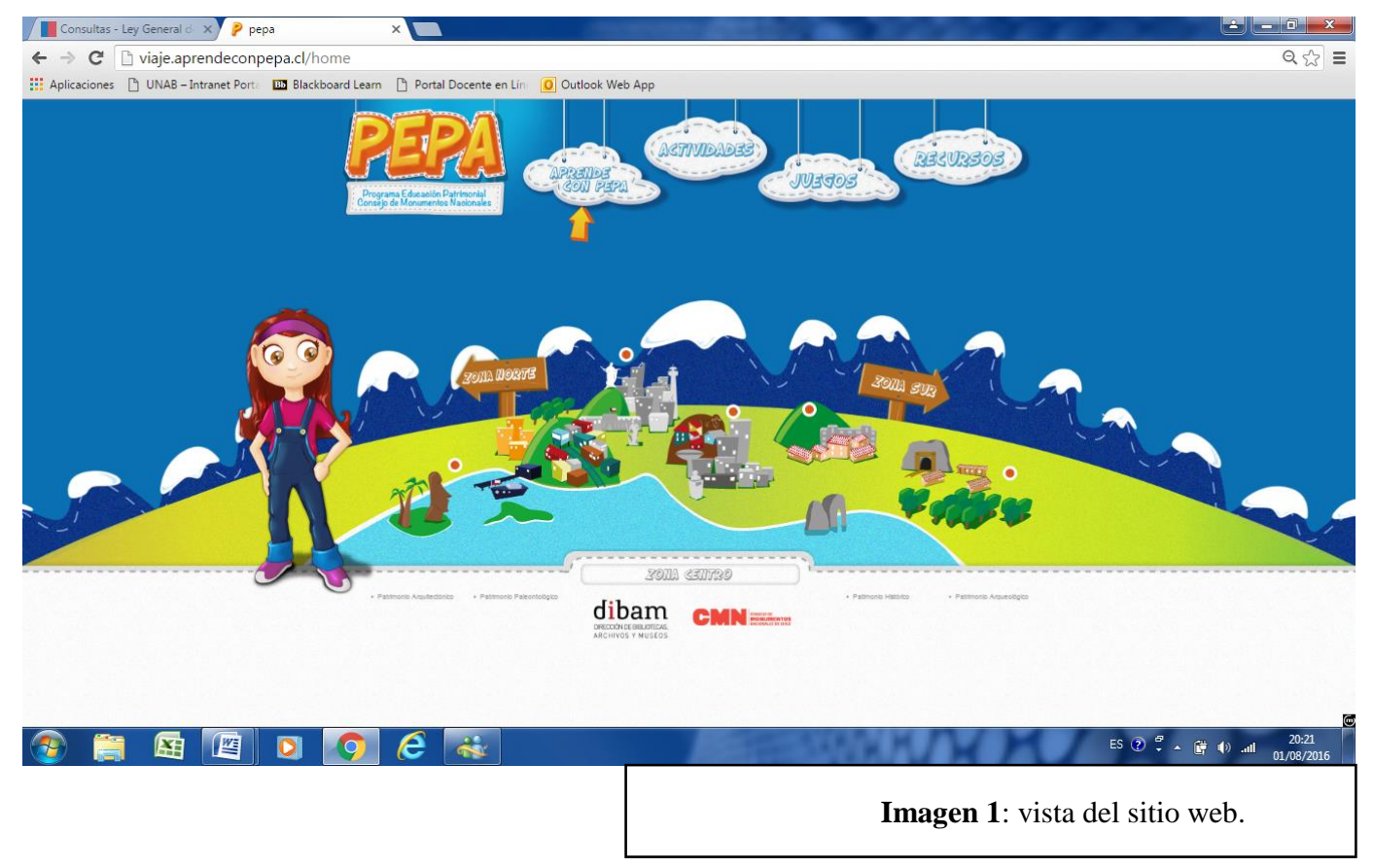

b) El programa llegará a la comunidad escolar a través de "PEPA visita tu Colegio". Esta actividad consiste en la ejecución de talleres y acciones pedagógicas sustentadas en una metodología de aprendizaje participativo, complementarias al currículo educativo nacional. Asociada a esta modalidad se incluye la entrega del Kit o "Material Didáctico PEPA" a los establecimientos que participen en el programa, con el fin de potenciar el trabajo sobre temáticas patrimoniales en el aula. 

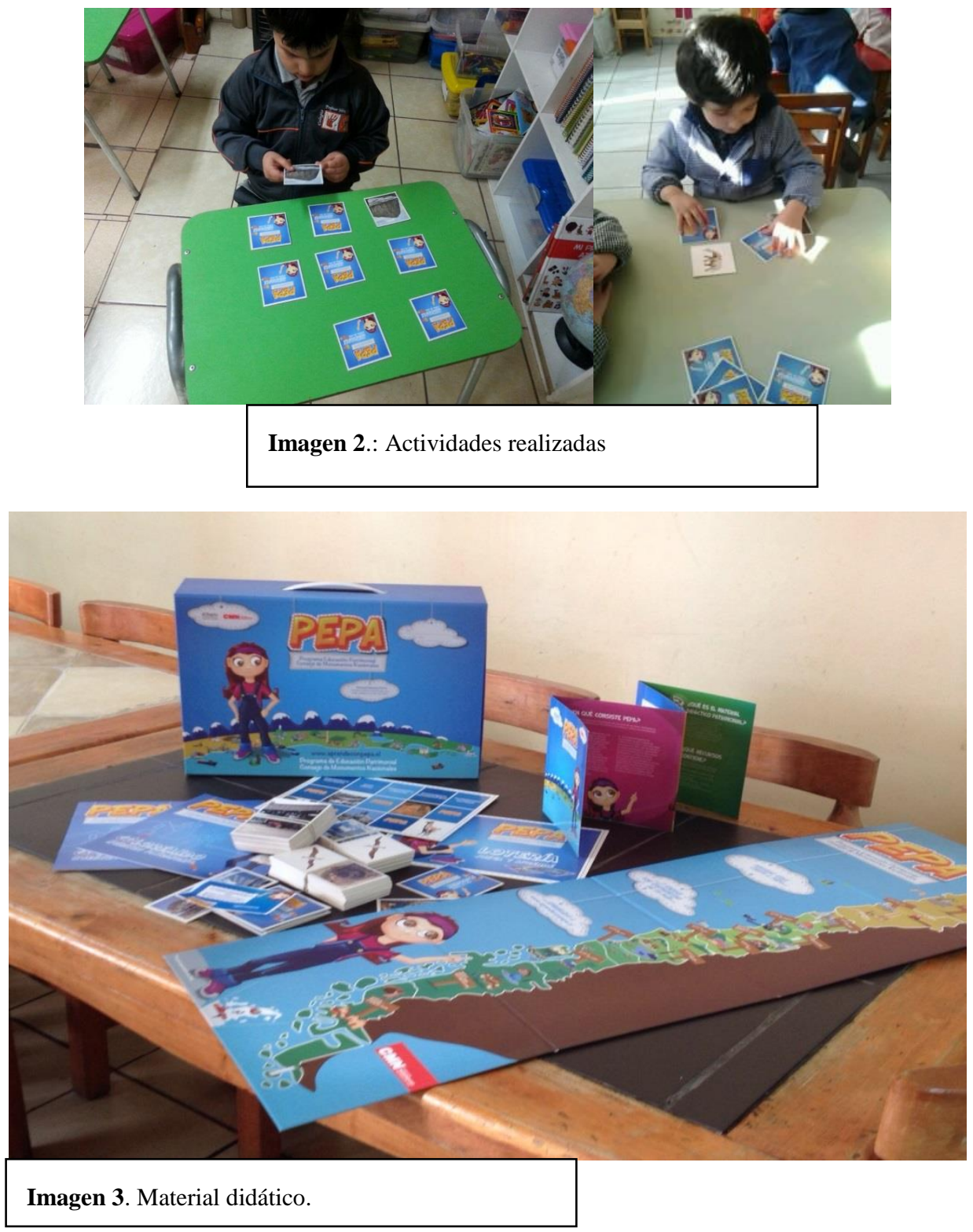

c) Capacitación Docente en Patrimonio y Material Educativo. Para ello se realizarán talleres formativos que permitan a los y las profesoras desarrollar herramientas y competencias interdisciplinarias para el trabajo educativo patrimonial utilizando el material didáctico patrimonial.

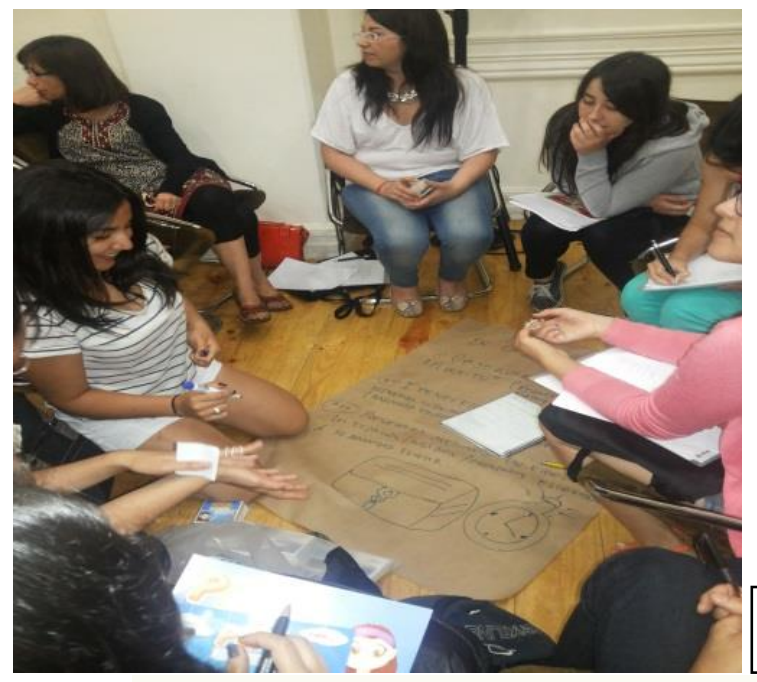

Imagen 4. Talleres docentes 


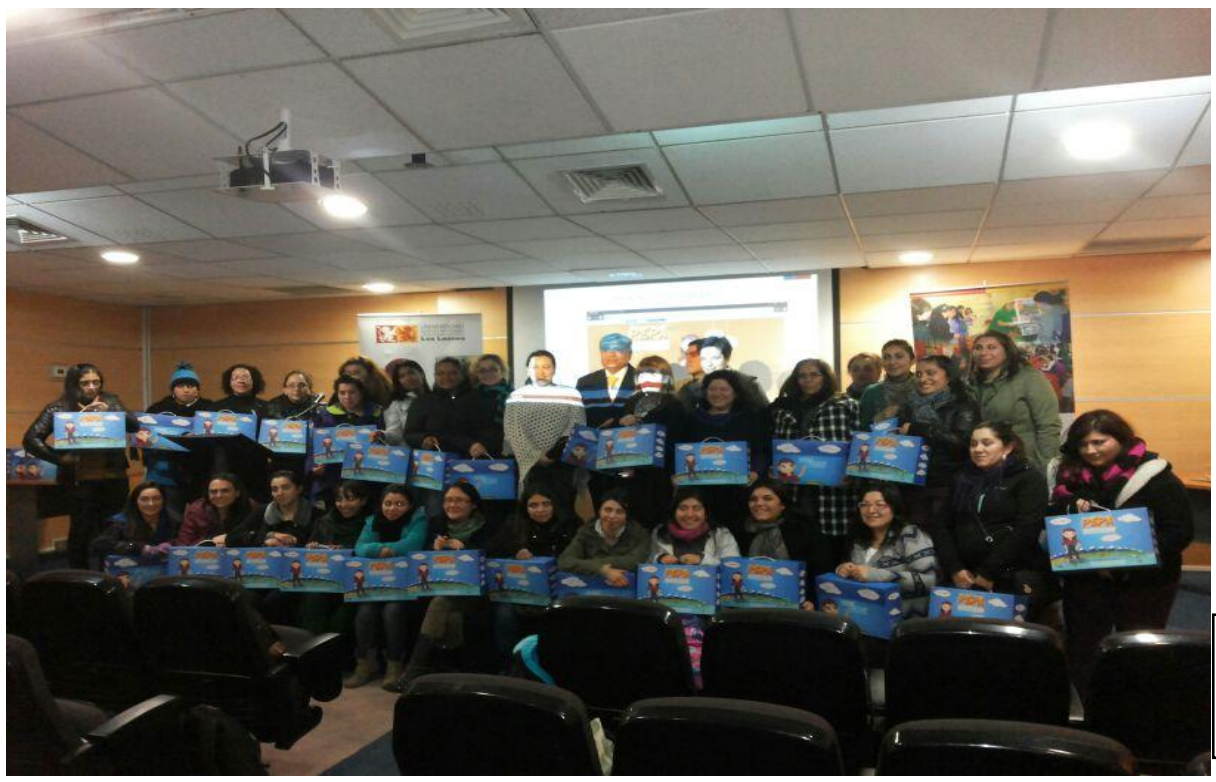

Imagen 5.

Entrega de material didáctico.

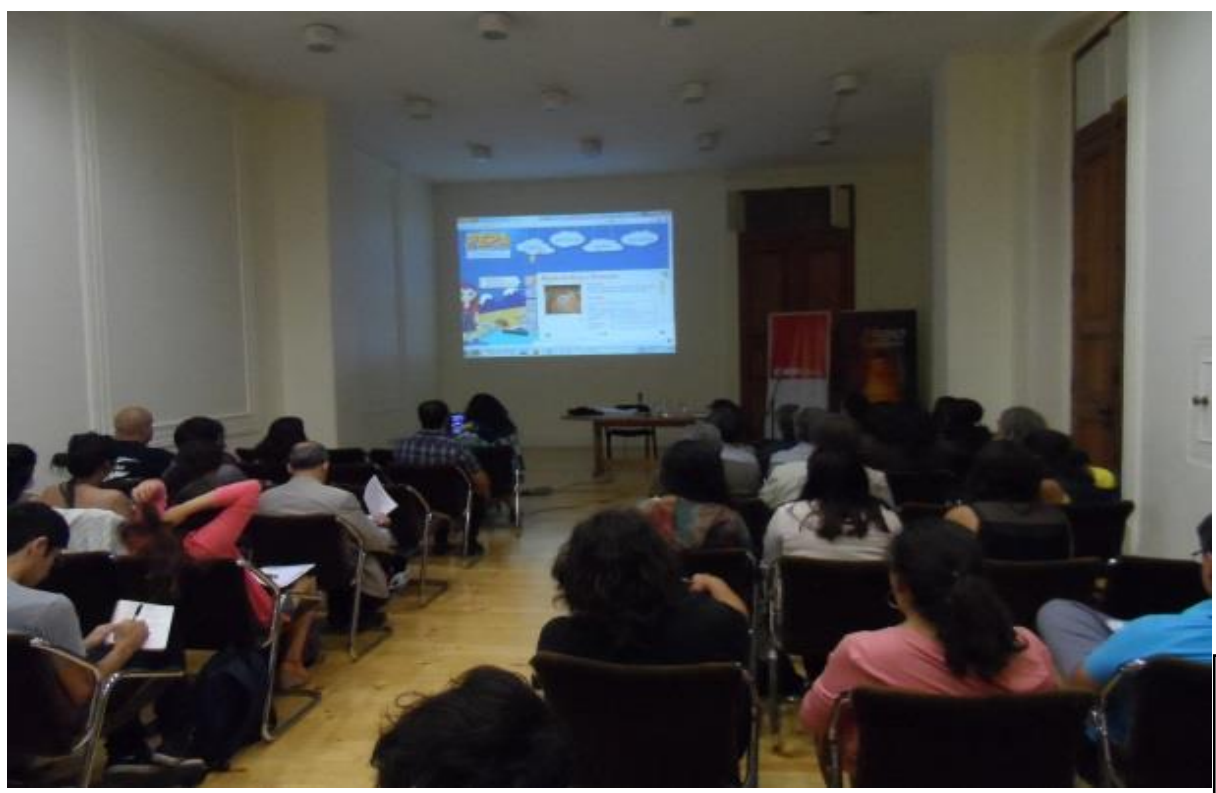

Imagen 6.

Jornada de capacitación.

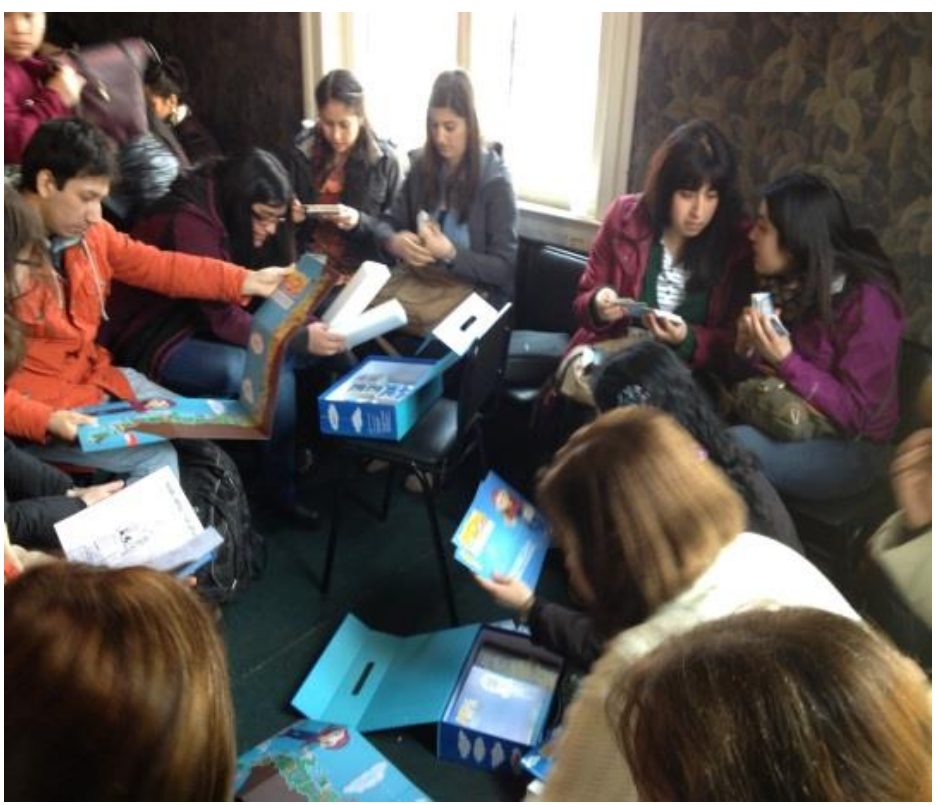

\section{Imagen 7.}

Capacitaciones realizadas. 


\section{¿QUÉ CONTIENE EL KIT O MATERIAL DIDÁCTICO PEPA?}

El material didáctico patrimonial es un conjunto de diversos recursos para potenciar el trabajo escolar en temáticas patrimoniales. Entrega herramientas conceptuales a los docentes y material concreto para uso en aula, destinado a niños y niñas de pre-básica y básica (primer ciclo).

1. "Guía de Apoyo Docente. Nociones generales sobre Patrimonio en Chile"

2. Recursos didácticos para el aula:

a) Lámina Ilustración Mapa de Chile

b) Set de juegos (Memorice-Dominó-Lotería)

c) Cuadernillo de trabajo: "Coloreando Nuestro Patrimonio"

d) Lámina Ilustración Mapa de Chile

\section{EVALUACIÓN}

Desde su puesta en marcha, en sus diferentes modalidades, han sido cientos de docentes y educadores capacitados en PEPA, así como escolares que a través de sus educadores han tenido contacto con el programa de educación patrimonial. Es por ello que resulta importante contar con información que nos permita reconocer el impacto que este ha tenido, así como una evaluación técnica de sus recursos, con el fin de realizar ajustes y mejoras al programa.

El alcance del proyecto en su primera etapa logró llegar a 700 docentes, distribuidos en ocho de las 15 regiones de nuestro país, esta evaluación se realizó con una muestra del 63\% del total de

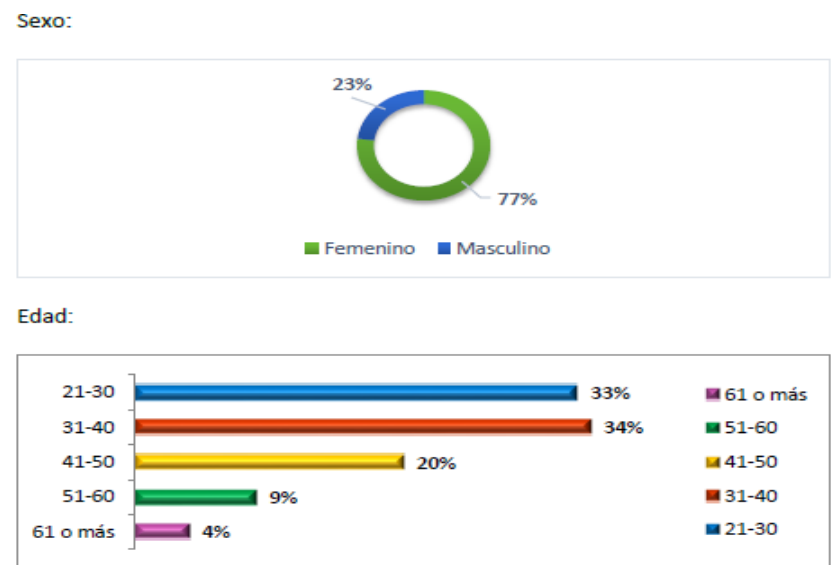

Ocupación:

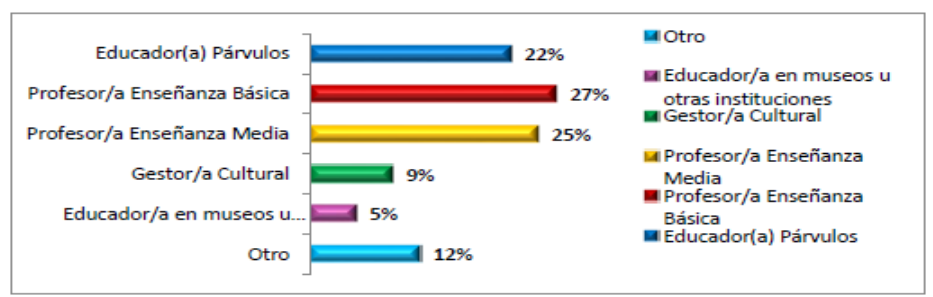

profesionales capacitados cuya representación se entrega a continuación:

Uno de los aspectos que nos interesaba evaluar era conocer la opinión de los docentes y educadores/as sobre las jornadas de capacitación a la que asistieron, la que tuvo por objetivo la presentación del Programa de Educación Patrimonial PEPA.

En estas jornadas se entregó una introducción al patrimonio histórico, cultural y natural del país, acercando a los participantes a un área de conoci- 
miento a la que no están habituados. Otra instancia importante estuvo dedicada a la exploración del maletín didáctico, resaltando sus cualidades como puerta de entrada para la introducción de los contendidos vinculados al patrimonio de una forma lúdica y creativa. Este primer acercamiento permitió a los asistentes reflexionar sobre la necesidad de conocer, comprender y proteger nuestro entorno y de transmitir estos valores desde la primera infancia.

\section{Claridad y adecuación de los contenidos} que se presentaron:

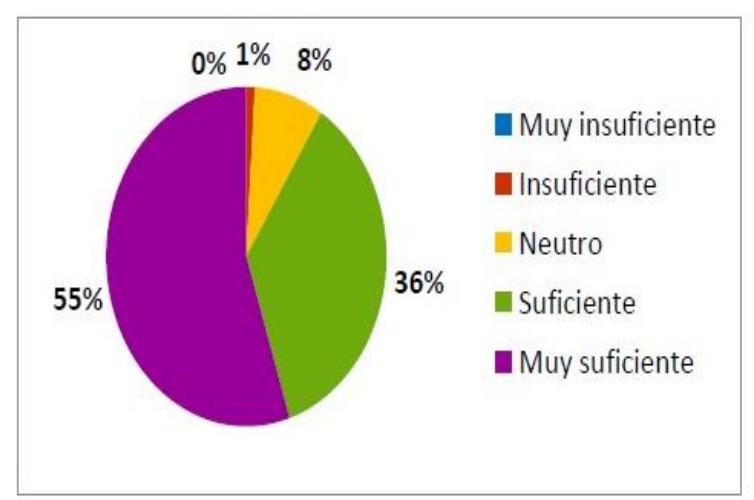

Utilidad de los contenidos para trabajo docente en aula:

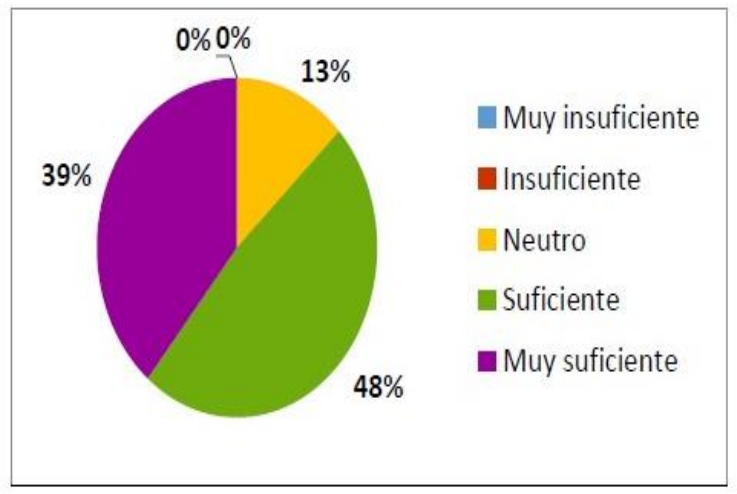

Los aspectos antes mencionados se ven ratificados en el alto porcentaje de encuestados que calificó la capacitación como "Muy suficiente o suficiente", lo que queda demostrado en cada uno de los aspectos evaluados: claridad y adecuación de los contenidos que se presentaron (91\%); utilidad de los contenidos para el trabajo docente en aula $(87 \%)$.

Otro ítem evaluado muestra un importante nivel de aceptación del material didáctico patrimonial por parte de los educadores, demostrado en el alto porcentaje de encuestados que calificó el material como "suficiente" en cada uno de los aspectos evaluados: claridad y utilidad de los conceptos del folleto de presentación del maletín (87\%); aspectos materiales y físicos del maletín (95\%); adecuación del set de juegos al nivel cognitivo de los alumnos/as de pre-básica y básica (87\%); y claridad y utilidad de los conceptos entregados por la guía de apoyo docente (92\%).

A continuación se muestra el desglose estadístico: 
Adecuación del set de juegos al nivel cognitivo de alumnos/as de pre-básica y básica.

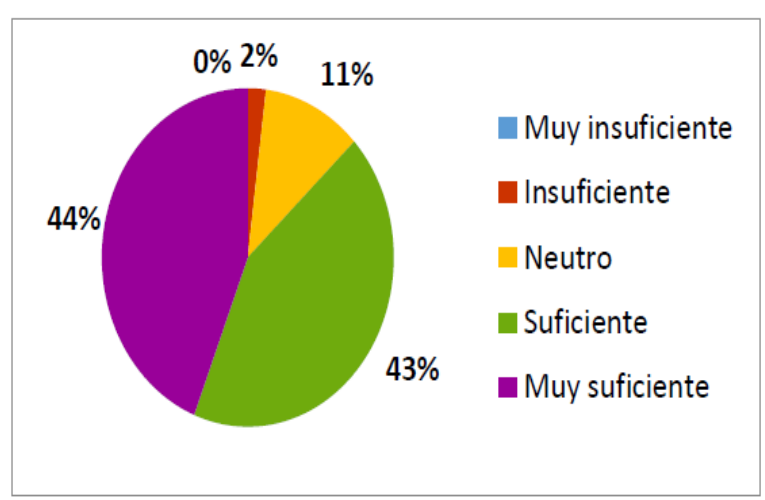

Claridad y utilidad de los conceptos entregados por la guía de apoyo docente.

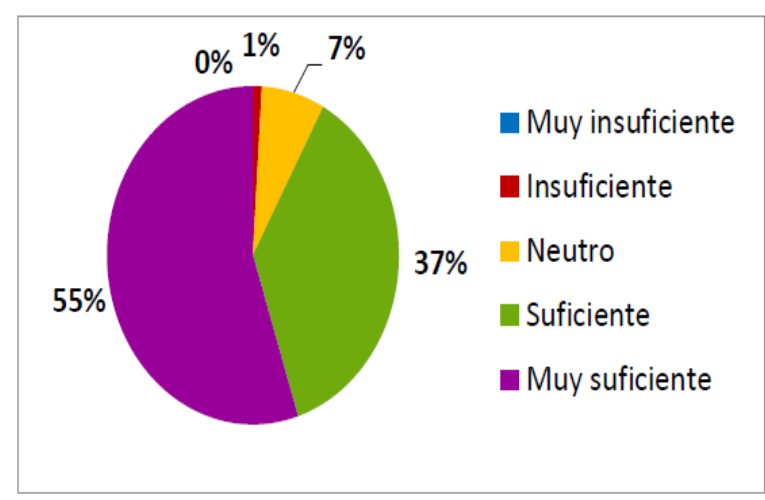

El tercer ítem evaluado fue el sitio www.aprendeconpepa.cl, el que tiene como propósito dotar a la comunidad escolar de contenidos informativos, didácticos y pedagógicos, para que sirva como complemento a todos los demás elementos que conforman el programa. Este punto tiene la intención de medir el cumplimiento de estos objetivos y la utilidad de sus contenidos.

Adecuación de los contenidos de la página web a las necesidades de la comunidad escolar (profesores y alumnos de pre-básica y primer ciclo).

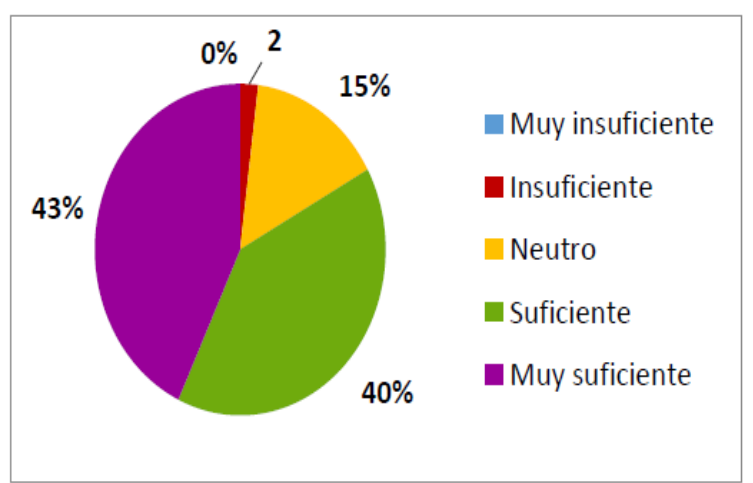

Claridad de los contenidos textuales sobre patrimonio y monumentos nacionales (definiciones, instrucciones, reseñas de monumentos, etc.)

Los resultados de la encuesta muestran un alto nivel de aceptación de la página web, demostrado en el porcentaje de encuestados que calificó como "suficiente" a cada uno de los aspectos evaluados: adecuación de los contenidos de la página web a las necesidades de la comunidad escolar (83\%); claridad de los contenidos textuales sobre patrimonio y monumentos nacionales(82\%); calidad gráfica de la página (80\%); calidad de los recursos pedagógicos disponibles (83\%); vinculación entre el objetivo de los juegos y la entrega de contenidos patrimoniales $(83 \%)$. 
Al realizar la consulta ¿En qué asignatura y/o ámbito de aprendizaje utilizaría los aspectos teóricos y recursos pedagógicos presentados en la capacitación? Las respuestas dijeron lo siguiente:

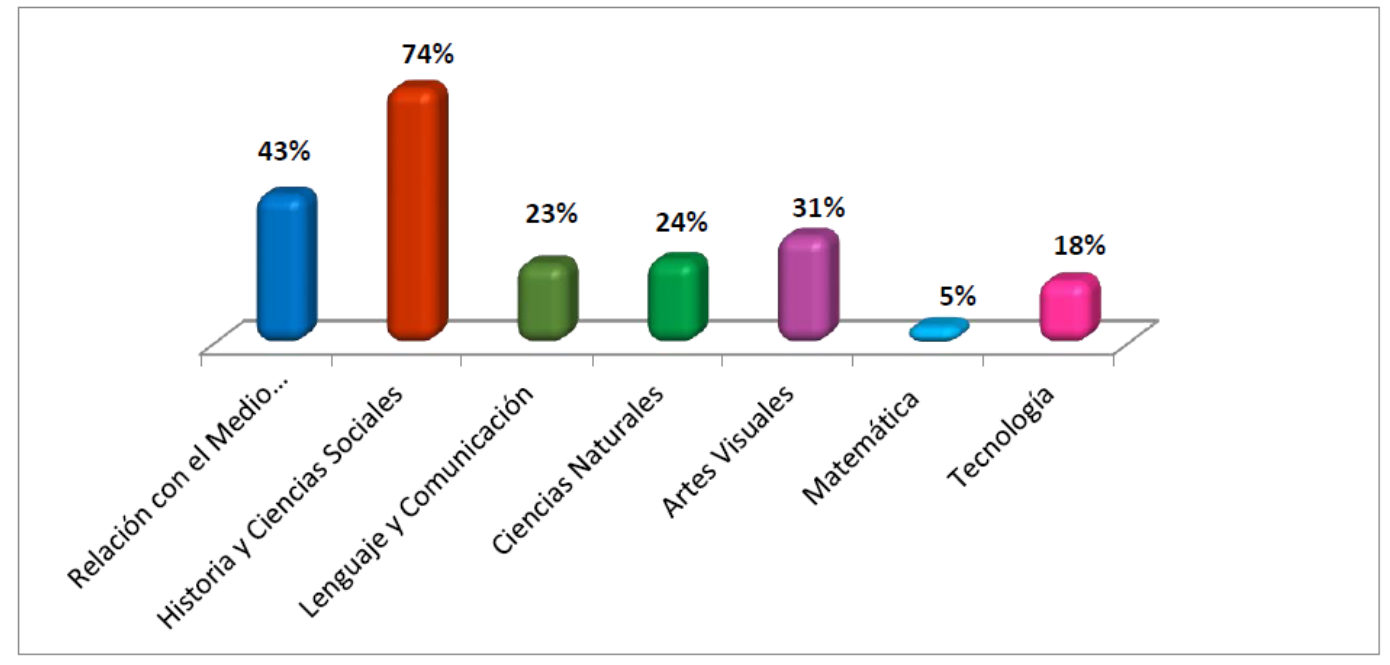

Una vez terminado el proceso de evaluación se entregaron las siguientes sugerencias para mejorar PEPA y sus componentes:

\section{Capacitación:}

- Realizar cursos de perfeccionamiento sobre patrimonio a docentes.

- Realizar más capacitaciones sobre PEPA donde se aborden los contenidos con más profundidad.

- Incluir capacitaciones en los establecimientos educacionales.

\section{Cobertura:}

- Ampliar la cobertura del programa de educación patrimonial a alumnos de Enseñanza Media

- Ampliar la difusión del programa para extenderlo a más establecimientos educacionales

\section{Material Didáctico Patrimonial:}

- Aumentar el número de maletines por curso.

- Aumentar el tamaño de las fichas para facilitar el trabajo grupal.

- Incluir referencias al patrimonio inmaterial.

- Complementar con visitas guiadas a circuitos patrimoniales.

Una vez realizada esta evaluación, se tomaron las primeras acciones, comenzando por el material didáctico patrimonial. Esta segunda versión toma las sugerencias que los docentes entregaron para que se transforme en un material más completo para el desarrollo de la educación patrimonial. 


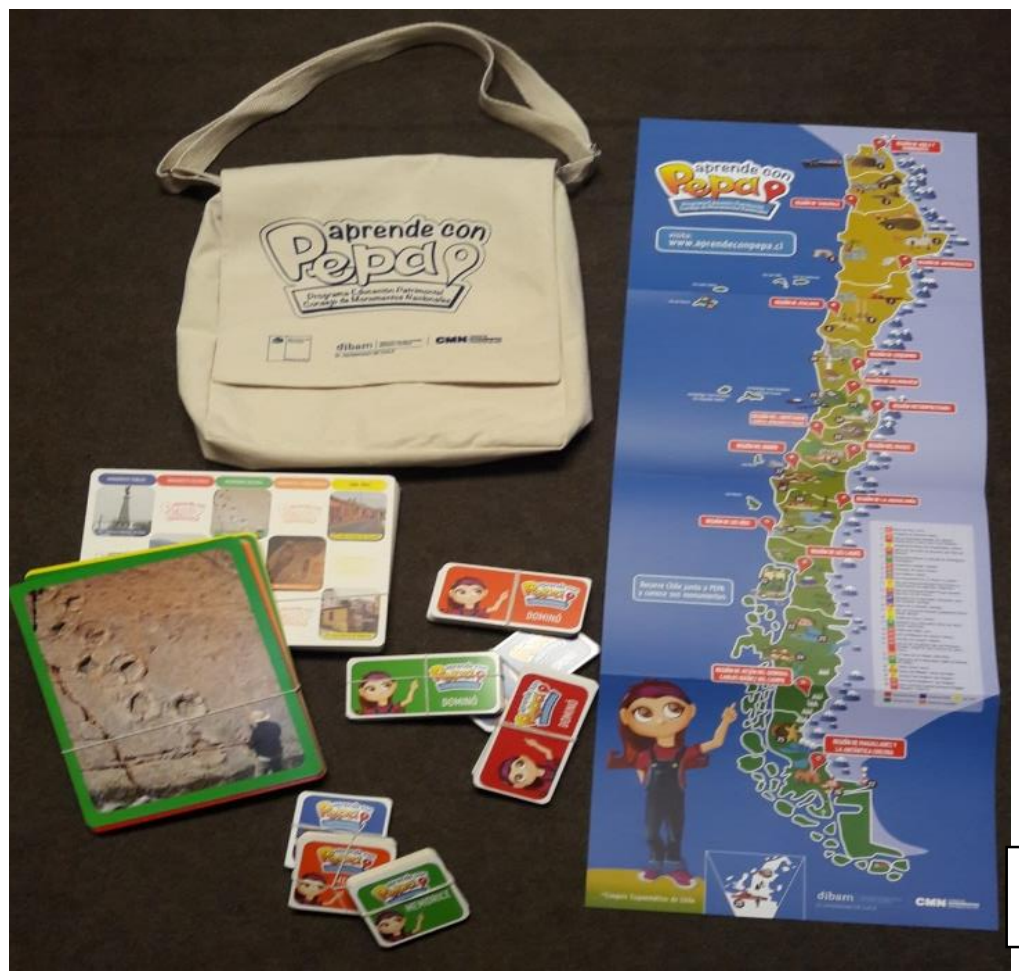

Imagen 8. Nuevo Material didáctico

\section{CONCLUSIÓN}

Estamos convencidas que el valor educativo que tiene el patrimonio cultural y natural reside en su aporte para la construcción de diversas identidades sociales y culturales, pues es un elemento mediador entre el pasado y el presente, además de una herramienta para el pensar futuro. Al mismo tiempo, el patrimonio es un elemento transversal en el currículo y, aunque su presencia en las prácticas dentro del aula no es regular ni sistemática, es un instrumento eficaz de aprendizaje para las distintas áreas del conocimiento.

Creemos, por la experiencia acumulada en estos años, que la mejor etapa para iniciar el reconocimiento y valoración del patrimonio cultural y natural, es en los primeros niveles de educación, con ello lograremos formar a ciudadanos consientes de la su importancia, además de desarrollar la valoración de lo que es nuestro, podremos estimular una pasión de largo aliento por la protección y conciencia de nuestro legado cultural, así como una relación reflexiva y crítica de cómo pensar y decidir el entorno. PEPA, está disponible y continúa implementando recursos de apoyo y perfeccionándose, para acercar a la comunidad escolar a la riqueza cultural del país. 


\section{REFERENCIAS}

CANTÓN, V. La Educación Patrimonial, como estrategia para la formación ciudadana. Correo del Maestro, n. 154, marzo, 2009. Disp.: 〈http://www.correodelmaestro.com/anteriores/2009/marzo/incert154.htm〉. Consultado: 20 febrero 2016.

HEVIA, R, HIRMAS, C y PEÑAFIEL, S. Patrimonio y cultura local en la escuela, Guía de experimentación e innovación pedagógica. Santiago: Oficina Regional de Educación de la Unesco. 2002. Disp.: 〈http://unesdoc.unesco.org/images/0015/001566/156618s.pdf >. Consultado: 15 de enero 2015.

EVALUACIÓN PROGRAMA DE EDUCACIÓN PATRIMONIAL PEPA, Licitación n. 4650-17-L115. Disp.: $\langle$ https://www.mercadopublico.cl/Home/BusquedaLicitacion>.

\section{RESUMEN}

El Programa de Educación Patrimonial "PEPA" del Consejo de Monumentos Nacionales-Chile, tiene por objetivo dotar al público escolar y docente de contenido informativo, didáctico y pedagógico respecto al patrimonio cultural y natural protegido. Este desarrolla 3 líneas de acción: www.aprendeconpepa.cl, PEPA en tu Colegio y Capacitación Docente con entrega de material. La iniciativa contribuye a generar un proceso de apropiación social de los valores patrimoniales desde el colegio. En tal sentido, se decide en el 2015 constatar la incidencia de PEPA y el impacto funcional del programa. Para la obtención de los datos se utilizaron métodos cuantitativos y cualitativos en personas capacitadas que permitieron una valoración fundamentada y técnicas aplicadas a grupos objetivos y controlados.

Palabras clave: Educación Patrimonial. PEPA. Capacitación docente.

\section{IMPLEMENTAÇÃO E AVALIAÇÃO DO PROGRAMA DE EDUCAÇÃO PATRIMONIAL - "PEPA"}

\section{RESUMO}

O Programa de Educação Patrimonial "PEPA" do Conselho de Monumentos Nacionais do Chile tem como objetivo dotar o público escolar e docente de conteúdo informativo, didático e pedagógico a respeito do patrimônio cultural e natural protegido pelo Estado. Este desenvolve três linhas de ação: www.aprendeconpepa.cl, PEPA no teu Colégio e Capacitação Docente com entrega de material. Esta iniciativa contribui para gerar um processo de apropriação social dos valores patrimoniais a partir do colégio. Nesse sentido, se decidiu, em 2015, constatar a incidência de "PEPA" e o impacto funcional do programa. Para a obtenção dos dados, se utilizaram métodos quantitativos e qualitativos em pessoas que passaram por processo de capacitação, as quais permitiram uma análise fundamentada e técnicas aplicadas a grupos objetivos e controlados.

Palavras-chave: Educação Patrimonial. PEPA. Formação Docente.

\section{IMPLEMENTATION AND EVALUATION OF THE HERITAGE EDUCATION PROGRAM - "PEPA"}

\section{ABSTRACT}

The Heritage Education Program "PEPA" of the Council of National Monuments Chile, aims to provide the student and teaching public informative, educational and pedagogical content regarding the cultural and natural heritage protected. This develops 3 lines of action: www.aprendeconpepa.cl, PEPA in your School and Teacher Training with delivery of material. The initiative contributes to a process of social appropriation of the heritage assets from school. Regarding this, in 2015 it was decided to verify the incidence of PEPA and the 
functional impact of the program. To obtain the information, quantitative and qualitative methods were used in trained people which allowed a based value and techniques applied to objective and controlled groups.

Keywords: Heritage Education, PEPA, Teacher Training

Submetido em: Ago 2016

Aprovado em: Dez 2016 